\title{
Vibration Analysis of a Cracked Beam Subjected to a Moving Mass
}

\author{
R.K. Behera and D.R.K. Parhi \\ Department of Mechanical Engineering, National Institute of Technology, Rourkela, 769008, Orissa, India
}

\author{
S.K. Sahu \\ Department of Civil Engineering, National Institute of Technology, Rourkela, 769008, Orissa, India
}

(Received 20 April 2004; accepted 18 October 2005)

\begin{abstract}
An analytical method is used to investigate the dynamic behaviour of a two-crack cantilever beam with a moving mass. In order to obtain the characteristic functions of a multi-cracked beam, the local stiffness matrices are taken into account. The Runge-Kutta numerical method has been used to solve the differential equations involved in analysing the dynamic deflection of a cracked cantilever beam with a moving mass. Comparisons are made between the dynamic deflection of a beam with a moving mass having no cracks and one with two-cracks, both of which are subjected to varying velocities and masses.
\end{abstract}

\section{Nomenclature}

$A \quad$ - beam cross-sectional area

$B \quad-$ width of the beam

$E \quad-$ modulus of elasticity of beam material

$g \quad-$ acceleration due to gravity

I - section moment of inertia of the beam

$L \quad-$ total length of the beam

$m \quad-$ mass per unit length of the beam

$M \quad-$ lumped mass of the moving body

$n \quad-$ integer variable (varies from 1 to $\infty$ )

$q \quad-$ integer variable (varies from 1 to $\infty$ )

$t \quad-$ time

$v \quad-$ velocity of the moving mass

$W \quad-$ height of the beam

$x \quad-$ distance of the point from the fixed end to the point of interest where deflection is desired

$y \quad-$ transverse dynamic deflection of the beam $a_{1}, a_{2} \quad-$ crack depths

$T_{n, t t}(t)=d^{2} T_{n}(t) / d t^{2}$

$V_{n}=\int_{0}^{L} Y_{n}^{2}(x) d x$

$Y_{n} \quad-$ eigen functions of the beam

$a_{1} / W, a_{2} / W$ - relative crack depths

$\beta=L_{1} / L \quad-$ relative crack position

$\gamma=L_{2} / L \quad-$ relative crack position

$\gamma_{n} \quad-$ eigenvalues

$\delta \quad-$ Dirac delta function

$\rho-\quad-$ mass density of the beam

$\omega_{n} \quad-$ natural circular frequency of the beam for the $n$-th mode

\section{INTRODUCTION}

Engineers have been investigating the potential hazard produced by the moving masses on structures for many several years. The dynamic response of structures carrying moving masses is a problem of widespread practical significance. In the early twentieth century, engineers such as Jeffcott ${ }^{1}$ managed to calculate the vibration response of simple struc- tures with a moving mass. The response characteristics of a beam subjected to a moving force were investigated by Florence $^{2}$, Steele ${ }^{3}$, Kenney ${ }^{4}$, and Smith $^{5}$. Stanisic and Hardin ${ }^{6}$, and Stanisic et al. $^{7}$ have developed a numerical-analytical method for determining the behaviour of beams with various boundary conditions and carrying a moving mass. Saigal ${ }^{6}$ has developed expressions for beam structures with the help of the Stanisic et al. theory ${ }^{7}$, which has a higher degree of practical significance. Later, Akin and Mofid ${ }^{8}$ analysed such problems for finite beams with moving loads using a differential equation. Parhi et al. ${ }^{9}$ have discussed the vibration analysis of a cantilever beam with a transverse crack using influence coefficients and stiffness elements at the crack section. None of these investigators have developed a theoretical approach for the dynamic deflection of a two-crack cantilever beam with a moving mass. In this paper, the local stiffness matrices at the cracked sections are taken into account for the dynamic deflection of a beam subjected to a moving mass.

\section{EQUATION OF MOTION}

The equation of motion for a uniform beam of mass $m$ subjected to a moving mass $M$, as shown in Fig. 1, neglecting damping, can be written as

$$
E I \frac{d^{4} y(x, t)}{d x^{4}}+m \frac{d^{2} y(x, t)}{d t^{2}}=\left[M g-M \frac{d^{2} y(\eta, t)}{d t^{2}}\right] \delta(x-\eta)
$$

where $m$ is the mass per unit length of the beam, $E$ is the modulus of elasticity of the beam, $I$ is the moment of inertia of the beam cross-section, $\eta$ is the distance considered from one end of the beam, $t$ is the time taken by the moving mass to travel a distance $\eta$ on the beam, $\delta$ is the Dirac delta function (see the Appendix), and $x$ is the distance of any arbitrary point $z$ on the beam.

The solution of Eq. (1) is assumed to be in a series form,

$$
y(x, t)=\sum_{n=1}^{\infty} Y_{n}(x) T_{n}(t),
$$

International Journal of Acoustics and Vibration, Vol. 10, No. 4, 2005

(pp 197-201) 